\title{
Herança da senescência retardada em milho
}

\author{
Emiliano Fernandes Nassau Costa ${ }^{(1)}$, Mateus Figueiredo Santos ${ }^{(1)}$, Gustavo Vitti Moro(1), Geovani Ferreira Alves(2) \\ e Cláudio Lopes de Souza Júnior ${ }^{(1)}$
}

\begin{abstract}
(1)Escola Superior de Agricultura Luiz de Queiroz, Departamento de Genética, Caixa Postal 83, CEP 13400-970 Piracicaba, SP E-mail: emilianonassau@hotmail.com,mfsantos@esalq.usp.br, gvmoro@esalq.usp.br, clsouza@esalq.usp.br (2)Sementes Adriana, Avenida Julia Maksoud, no 145, Bairro Monte Castelo, CEP 79011-100 Campo Grande, MS. E-mail: geovani_alves@yahoo.com.br
\end{abstract}

Resumo - O objetivo deste trabalho foi estudar a herança da senescência retardada em milho. Foram realizados cruzamentos dialélicos parciais entre 50 linhagens e cinco testadores. Os 250 cruzamentos resultantes, além de seis híbridos comerciais utilizados como testemunhas, foram avaliados em oito ambientes, no delineamento látice simples 16x16, com duas repetições por ambiente. Os cruzamentos dialélicos foram analisados utilizando o método 4 do modelo 1 de Griffing, adaptado para múltiplos ambientes. A contribuição da capacidade geral de combinação (CGC) para a expressão do caráter "stay-green" (69,06\%) foi maior que a da capacidade específica de combinação (CEC) (30,94\%), evidenciando que os efeitos aditivos são mais importantes que os efeitos não aditivos na expressão deste caráter. Tanto a CGC como a CEC interagiram significativamente com o ambiente, indicando que a seleção para este caráter deve ser realizada com base nas médias de experimentos em diversos ambientes.

Termos para indexação: Zea mays, CEC, CGC, cruzamento dialélico, stay-green.

\section{Inheritance of the delayed senescence in maize}

\begin{abstract}
The objective of this research was to study the inheritance of delayed senescence in maize. Partial diallel crosses among 50 inbred lines and five testers were made. The 250 crosses, along with six commercial hybrids used as checks, were evaluated at eight environments in lattices 16x16 with two replications per environment. The diallel crosses were analyzed following the method 4 model 1 of Griffing, extended to multiple environments. The contribution of the general combining ability (CGA) for the expression of the stay-green trait $(69.06 \%)$ was greater than the specific combining ability (SCA) (30.94\%), showing that additive effects are more important than non-additive effects for the expression of this trait. Both GCA and SCA interacted significantly with the environments, indicating that the selection for this trait should be based on the means across environments.
\end{abstract}

Index terms: Zea mays, SCA, GCA, diallel crossing, stay-green.

\section{Introdução}

Edmeades et al. (1999) relatam que o milho cultivado na maioria das regiões tropicais sofre uma redução de até $50 \%$ na produção devido ao estresse provocado por secas. De acordo com esses autores, o melhoramento genético pode reduzir de 15 a $25 \%$ essas perdas. Chapman \& Edmeades (1999), a partir de resultados obtidos em um estudo para tolerância à seca, realizado com milho, sugerem que os genótipos com elevada senescência retardada ("stay-green"), alta prolificidade e reduzido intervalo entre florescimentos são menos afetados por estresses hídricos.

Em programas de melhoramento genético de cereais, os genótipos são considerados "stay-green" quando as folhas e colmos permanecem verdes após o enchimento dos grãos. Dessa forma, após o enchimento dos grãos, a fotossíntese se prolonga fornecendo carboidratos para colmos, folhas e raízes e, consequientemente, as plantas apresentam maior resistência a estresses bióticos e abióticos (Tollenar \& Wu, 1999; Jiang et al., 2004; Carmo et al., 2007). Além disso, os genótipos com "stay-green" apresentam maior resistência ao acamamento, pois os carboidratos do colmo extraídos para o enchimento dos grãos são repostos, o que reduz o quebramento de colmos e aumenta a resistência às doenças de raízes (Duvick \& Cassman, 1999).

A herança do caráter "stay-green" tem sido pouco estudada na cultura do milho e a variação genética existente para senescência foliar vem sendo 
empiricamente explorada pelos programas de melhoramento (Thomas \& Smart, 1993). A base genética do "stay-green" tem sido estudada em diversas culturas como arroz, feijão e trigo (Jiang et al., 2004; Carmo et al., 2007; Joshi et al., 2007), porém, em milho, os dados acerca da base genética do caráter "stay-green" ainda são raros, havendo necessidade de maiores estudos para melhor elucidação do seu controle genético.

Este trabalho teve como objetivo estudar a herança do caráter "stay-green" em milho, por meio de cruzamentos dialélicos.

\section{Material e Métodos}

Foram utilizadas 55 linhagens endogâmicas de milho de diversas origens, das quais cinco eram linhagens-elite previamente selecionadas para serem utilizadas como testadoras. As 55 linhagens eram oriundas de diversas populações (IG-1, IG-2 e CMS-05), de alguns híbridos comerciais (BR-201, C-601, C-606, HS-1 e XL-560) e de progênies de meios-irmãos interpopulacionais (BR-105 x BR-106). Todas as linhagens foram obtidas no programa de melhoramento de milho do Departamento de Genética, da Escola Superior de Agricultura Luiz de Queiroz (Esalq), em Piracicaba, e apresentavam níveis de endogamia de pelo menos seis gerações de autofecundações. As linhagens, por serem de origens distintas, diferiram umas das outras quanto a diversos caracteres, inclusive quanto ao caráter "staygreen".

Foram realizados cruzamentos dialélicos parciais, em que as cinco linhagens-elite utilizadas como testadoras foram cruzadas com as demais 50 linhagens, no ano agrícola de 2003/2004, em um total de 250 cruzamentos. Para a obtenção dos cruzamentos, as 50 linhagens de um grupo foram plantadas pareadas com as cinco linhagens do outro grupo. As parcelas foram constituídas de uma linha de $6 \mathrm{~m}$ com 30 plantas, espaçadas $0,80 \mathrm{~m}$ entre linhas e a $0,20 \mathrm{~m}$ entre plantas. Para garantir a coincidência do florescimento, tanto masculino como feminino, os testadores foram plantados em três épocas distintas: cinco dias antes; no mesmo dia; e cinco dias após as demais linhagens. Os cruzamentos foram realizados manualmente, com o uso de cruzamentos recíprocos. Na colheita, os cruzamentos recíprocos de cada par foram colhidos em conjunto e misturados para gerar quantidade de sementes suficientes para avaliação em experimentos com repetições. Foram utilizados seis híbridos comerciais como testemunhas.
Os 256 tratamentos obtidos com os cruzamentos foram avaliados em oito ambientes, entre os anos agrícolas 2004/2005 e 2006/2007. A combinação ano agrícola $\mathrm{x}$ local correspondeu a um ambiente. Foram utilizados os seguintes ambientes: Estação Experimental Departamento de Genética da Esalq, nos anos agrícolas 2004/2005, 2005/2006 e 2006/ 2007; Estações Experimentais Caterpillar e Areão, nos anos agrícolas 2004/2005 e 2005/2006; e Estação Experimental Anhembi, no ano agrícola 2005/2006.

O delineamento experimental utilizado foi o látice simples 16x16 com duas repetições. As parcelas foram constituídas por linhas de $4 \mathrm{~m}$ de comprimento, espaçadas $0,80 \mathrm{~m}$ entre linhas e $0,20 \mathrm{~m}$ entre plantas. Foram plantadas 50 plantas por parcela, e realizado o desbaste aos 30 dias após o plantio, deixando-se um estande ideal de 20 plantas por parcela, equivalente a um estande médio de 62.500 plantas ha-1.

Os caracteres avaliados foram "stay-green" $\mathrm{e}$ florescimento feminino. O caráter "stay-green" foi avaliado após a maturação fisiológica dos grãos, aos 120 dias após o plantio, por meio de uma escala de notas de 1 a 5 , em que 1 referiu-se às plantas com todas as folhas acima da espiga e pelo menos duas folhas verdes abaixo da espiga; nota 2 , às plantas em que todas as folhas acima da espiga estivessem verdes; nota 3 , às plantas em que duas folhas acima da espiga estivessem secas e as demais verdes; nota 4 , às plantas em que duas folhas no ápice da planta estivessem verdes; e nota 5 , às plantas em que todas as folhas estavam secas. Foram avaliadas cinco plantas competitivas por parcela, e a média de suas notas foi utilizada nas análises.

Para florescimento feminino, considerou-se o número de dias do plantio até que $50 \%$ das plantas na parcela apresentassem estilo-estigmas.

As análises de variâncias individuais, conjunta e dialélica foram realizadas com o programa computacional SAS versão 8.2, módulo PROC GLM. Inicialmente, foram realizadas análises individuais de cada ambiente, por meio de um modelo misto, que considerava o efeito de tratamentos fixo e os demais aleatórios. As médias de cada parcela foram corrigidas, por covariância, com o número de dias de cada parcela para o caráter florescimento feminino. Após as análises individuais, foi realizada a análise de variância conjunta envolvendo os oito 
ambientes de avaliação, em que o modelo foi considerado misto, com todos os efeitos aleatórios, excetuando-se a média e tratamento. Para realizar esta análise, foram utilizadas as médias ajustadas de cada ambiente. No entanto, como essas médias foram obtidas com recuperação da informação do resíduo intrabloco, em cada uma delas foi necessário calcular o erro efetivo. As médias dos quadrados médios dos erros foram utilizadas para compor o erro efetivo médio da análise conjunta, conforme Cochram \& Cox (1957).

Com base nas esperanças dos quadrados médios da análise de variância conjunta, foram estimadas a variância fenotípica $\left(\hat{\sigma}_{\overline{\mathrm{F}}}^{2}\right)$ da interação cruzamento ambiente $\left(\hat{\sigma}_{\mathrm{CXA}}^{2}\right)$ e do erro $\left(\hat{\sigma}_{\mathrm{E}}^{2}\right)$. Os efeitos genéticos foram considerados fixos e por isso foram estimados o componente quadrático genético de cruzamentos $\left(\hat{V}_{C}\right)$ e o coeficiente de determinação genotípico $\left(R_{C}^{2}\right)$.

A análise de variância do dialelo foi realizada utilizando-se as médias ajustadas do caráter "staygreen" de cada uma das análises individuais dos 250 cruzamentos, excetuando-se as testemunhas. A análise de variância do dialelo foi realizada de acordo com o método 4 do modelo 1 de Griffing (1956), adaptado para dialelos parciais em múltiplos ambientes (Ferreira et al., 1993; Wu \& Matheson, 2000, 2001; Zhang \& Kang, 2003). O modelo matemático utilizado foi:

$\mathrm{Y}_{\mathrm{rs}}=\mu+\mathrm{a}_{1}+\mathrm{g}_{\mathrm{r}}+\mathrm{g}_{\mathrm{s}}+\mathrm{s}_{\mathrm{rs}}+(\mathrm{ag})_{\mathrm{rl}}+(\mathrm{ag})_{\mathrm{sl}}+(\mathrm{as})_{\mathrm{rs}}+\overline{\mathrm{e}}_{\mathrm{rsl}}$ em que: $Y_{\text {rsl }}$ é o valor médio observado da combinação híbrida entre a r-ésima linhagem $\left(\mathrm{g}_{1}\right)$ com a s-ésima linhagem $\left(\mathrm{g}_{2}\right)$ no ambiente $1 ; \mu$ é a média geral do caráter; $a_{1}$ é o efeito do ambiente 1 $(1=1,2,3, \ldots, 8) ; \mathrm{g}_{\mathrm{r}}$ é o efeito da capacidade geral de combinação da r-ésima linhagem $\left(\mathrm{g}_{1}\right)(\mathrm{r}=1,2,3, \ldots, 5)$; $\mathrm{g}_{\mathrm{s}}$ é o efeito da capacidade geral de combinação da s-ésima linhagem $\left(\mathrm{g}_{2}\right)(\mathrm{s}=1,2,3 \ldots, 50) ; \mathrm{s}_{\mathrm{rs}}$ é o efeito da capacidade específica de combinação entre a linhagem $\mathrm{r}\left(\mathrm{g}_{1}\right)$ e a linhagem s $\left(\mathrm{g}_{2}\right)$; $(\mathrm{ag})_{\mathrm{rl}}$ é o feito da interação entre a capacidade geral de combinação da r-ésima linhagem $\left(\mathrm{g}_{1}\right)$ e o efeito de ambientes; $(\mathrm{ag})_{\mathrm{sl}}$ é o efeito da interação entre a capacidade geral de combinação da s-ésima linhagem $\left(\mathrm{g}_{2}\right)$ e o efeito de ambientes; (as) rsl $_{\text {rs }}$ o efeito da interação entre a capacidade específica de combinação das linhagens $r\left(g_{1}\right)$ e as linhagens s $\left(g_{2}\right)$ e o efeito de ambientes; $\overline{\mathrm{e}}_{\text {rsl }}$ é o erro experimental médio.
$\mathrm{Na}$ análise de variância, a soma de quadrados de cruzamentos foi desdobrada em capacidade geral de combinação (CGC) das linhagens do grupo $1\left(\mathrm{~g}_{1}\right)$ e CGC das linhagens do grupo $2\left(\mathrm{~g}_{2}\right)$ e em capacidade específica de combinação (CEC). A seguir, a soma de quadrados de Cruzamentos x Ambientes foi desdobrada em CGC do grupo 1 x Ambientes, CGC do grupo 2 x Ambientes e CEC x Ambientes. Para obter a proporção da contribuição dos efeitos aditivos e dos não aditivos, foram estimadas a contribuição relativa da capacidade geral de combinação e a contribuição relativa da capacidade específica de combinação, que foram obtidas conforme Santos et al. (2007). As estimativas das capacidades gerais de combinação das linhagens do grupo 1, das linhagens do grupo 2 e das capacidades específicas de combinação foram obtidas conforme descrito por Vencovsky \& Barriga (1992).

\section{Resultados e Discussão}

Nas análises de variância individuais do caráter "staygreen", a relação entre o maior e o menor valor da variância do erro experimental foi de 2,55. Portanto, as variâncias do erro experimental podem ser consideradas homogêneas.

$\mathrm{Na}$ análise conjunta de variância do caráter "staygreen", foram detectadas diferenças significativas para todas as fontes de variação. A significância observada nos ambientes ocorreu em razão da diversidade de condições edafoclimáticas entre os oito ambientes estudados. A diferença significativa entre os tratamentos, apontada pela análise conjunta, indica a presença de variabilidade genética entre os tratamentos, que também foi observada nas análises individuais. Essa variabilidade era esperada, uma vez que as 55 linhagens envolvidas nos cruzamentos eram de origens diversas. A significância dos cruzamentos e de seus desdobramentos entre as cinco linhagens testadoras indicou a presença de variabilidade genética entre os cruzamentos resultantes, a existência de variabilidade genética para cada cruzamento, e que pelo menos um dos tipos de cruzamento diferia dos demais. Foi possível observar, também, que as testemunhas diferiam geneticamente entre si e que, em média, os cruzamentos e as testemunhas apresentavam valores diferentes para o caráter "stay-green".

Com relação às médias obtidas na análise conjunta, a média geral para o caráter "stay-green" foi de 3,51. 
A média apresentada pelas testemunhas foi de 3,11, e variou de 2,39 a 3,61. A média dos cruzamentos foi de 3,52 , com variação de 2,32 a 4,48. Observando-se que a nota 1 era atribuída à planta com "stay-green" e a nota 5 à planta sem "stay-green", as testemunhas obtiveram, em média, uma performance superior aos cruzamentos. Porém, quando as médias de cada cruzamento foram comparadas individualmente à média de cada testemunha, notou-se que $14,8 \%$ delas foram inferiores. Para exemplificar, pode-se citar o cruzamento da linhagem $26 \mathrm{com}$ a linhagem testadora 5, em que foi obtida média de 2,32, enquanto a média mínima apresentada pelas testemunhas foi de 2,39.

A variabilidade genética entre os cruzamentos foi elevada, bem próxima à estimativa da variância fenotípica, indicativo de que as diferenças entre os cruzamentos estavam relacionadas, principalmente, à sua constituição genética. Porém, a variância da interação cruzamento $\mathrm{x}$ ambiente também foi relativamente alta, representando $65,94 \%$ da estimativa da variabilidade genética. Isto indica que os cruzamentos sofreram influência do ambiente na sua expressão fenotípica. $O$ valor do coeficiente de determinação genotípico $\left(\mathrm{R}_{\mathrm{C}}^{2}\right)$ foi de $82,08 \%$, tendo sido calculado utilizando a variância fenotípica entre as médias dos cruzamentos nos diversos ambientes. Valores semelhantes foram encontrados por Câmara (2006) e por Bekavac et al. (2007). Quando baseado na média de parcela sem repetição, o valor do $R_{C}^{2}$ caiu para $26,07 \%$. O coeficiente de determinação genotípico com valor de $82,08 \%$ permite dizer que há alta confiabilidade dos valores fenotípicos em representar os valores genotípicos (Yokomizo \& Vello, 2000). Porém, como houve um elevado valor da $\hat{\sigma}_{\mathrm{CxA}}^{2}$ e do $\hat{\sigma}_{\mathrm{E}}^{2}$, o "stay-green" revelou ser um caráter complexo (Carmo et al., 2007). Isto implica que, para se realizar uma seleção eficiente, esta deve ser realizada com base nas médias dos cruzamentos avaliados em experimentos com repetições e em diversos ambientes (Tabela 1).

O resultado da análise dialélica detectou diferenças significativas $(\mathrm{p}<0,01)$ para as capacidades gerais de combinação das linhagens testadoras $\left(\mathrm{CGC}_{\mathrm{T}}\right)$ e para as capacidades gerais de combinação das linhagens em avaliação $\left(\mathrm{CGC}_{\mathrm{LI}}\right)$, indicando, desta forma, que as linhagens contribuíram diferentemente para os cruzamentos em que estiveram envolvidas. Também foram detectadas diferenças significativas para as capacidades específicas de combinação $\left(\mathrm{CEC}_{\mathrm{TxLI}}\right)$, indicando que as combinações híbridas apresentavam performances diferentes das esperadas somente pelos efeitos das capacidades gerais de combinação $\left(\mathrm{CGC}_{\mathrm{T}}\right.$ e $\left.\mathrm{CGC}_{\mathrm{LI}}\right)$ e que há elevada divergência genética entre as linhagens. Os efeitos das interações das capacidades gerais de combinação $\mathrm{CGC}_{\mathrm{T}} \mathrm{x}$ Ambientes, $\mathrm{CGC}_{\mathrm{LI}} \mathrm{x}$ Ambientes e das capacidades específicas de combinação $\mathrm{CEC}_{\mathrm{TxLI}} \mathrm{x}$ Ambientes foram significativas, indicando que os efeitos das capacidades gerais de combinação $\left(\mathrm{CGC}_{\mathrm{T}}\right.$ e $\left.\mathrm{CGC}_{\mathrm{LI}}\right)$ apresentaram alterações nos ambientes em que os cruzamentos foram avaliados. $\mathrm{O}$ efeito da interação das capacidades específicas de combinação CEC $_{\mathrm{TxLI}}$ $\mathrm{x}$ Ambientes, que também foi significativa, permite inferir que estas não se mostraram estáveis nos ambientes em que foram avaliadas. Portanto, a expressão do caráter "stay-green" depende do ambiente de avaliação, o que pode dificultar o processo de seleção, conforme resultados obtidos por Carmo et al. (2007).

A contribuição da percentagem relativa da capacidade geral de combinação foi de $69,06 \%$, enquanto a percentagem relativa da capacidade específica de combinação foi de $30,94 \%$, o que evidencia maior importância relativa dos efeitos

Tabela 1. Estimativas da variância fenotípica $\left(\hat{\sigma} \frac{2}{\mathrm{~F}}\right)$, interação cruzamentos x ambientes $\left(\hat{\sigma}_{\mathrm{CxA}}^{2}\right)$, erro experimental $\left(\hat{\sigma}_{\mathrm{E}}^{2}\right)$, estimativa da variabilidade genética entre cruzamentos $\left(\hat{\mathrm{V}}_{\mathrm{C}}\right)$, coeficiente de determinação genotípico entre cruzamentos $\left(\mathrm{R}_{\mathrm{C}}^{2}\right)$, e intervalos de confiança $(\mathrm{IC})^{(1)}$ correspondentes para o caráter "stay-green" (SG).

\begin{tabular}{cc}
\hline Parâmetro & $\mathrm{SG}\left(\mathrm{x} 10^{2}\right)$ \\
\hline$\hat{\sigma}_{\bar{F}}^{2}$ & 16,21 \\
$\mathrm{IC}$ & {$[13,70 ; 19,48]$} \\
$\hat{\sigma}_{C x A}^{2}$ & 8,77 \\
$\mathrm{IC}$ & {$[7,41 ; 10,54]$} \\
$\hat{V}_{C}$ & 13,30 \\
$\mathrm{IC}$ & {$[10,85 ; 16,66]$} \\
$\hat{\sigma}_{E}^{2}$ & 28,95 \\
\hline$R_{C}^{2}$ & 82,08 \\
$\mathrm{IC}$ & {$[78,22 ; 85,05]$} \\
\hline
\end{tabular}

(1) Intervalos de confiança com $\alpha=95 \%$ de probabilidade. 
gênicos aditivos sobre os não aditivos (dominância e epistasia) na expressão fenotípica do caráter "stay-green"; entretanto a alta significância dos efeitos da $\mathrm{CEC}_{\mathrm{TxLI}}$ também implica a contribuição de efeitos gênicos não aditivos na expressão da variância fenotípica entre os cruzamentos (Tabela 2), concordando com resultados obtidos em outras culturas como girassol e trigo (Cukadar-Olmedo et al., 1997; Joshi et al., 2007).

As estimativas das capacidades gerais de combinação do grupo de linhagens testadoras (g1) variaram de -0,21 para a linhagem testadora 5(L-46-10D) a 0,17 para a linhagem testadora 4 (L-49-02D). Esses valores concordam com as médias obtidas entre cada cruzamento, em que os cruzamentos com a linhagem testadora 5 foram os que obtiveram média mais baixa $(3,31)$, enquanto a média mais alta foi obtida nos cruzamentos com a linhagem testadora $4(3,69)$. As linhagens testadoras que contribuíram com a redução das notas e, conseqüentemente com o aumento da expressão do caráter, foram as linhagens 1 (L-08-05F), com estimativa de -0,11, e 5 (L-46-10D), com estimativa de $-0,21$. Com relação às capacidades gerais de combinação do outro grupo de linhagens, $62 \%$ das 50 estimativas foram significativas ou altamente significativas. Entre as estimativas que foram significativas ou altamente significativas, 45,2\% contribuíram com o aumento da expressão do caráter, ou seja, $28 \%$ do total de estimativas. As linhagens do

Tabela 2. Quadrados médios, significâncias, percentagens relativas da capacidade geral de combinação (CGC), capacidade específica de combinação (CEC) da análise dialélica e coeficiente de variação $(\mathrm{CV})$ para o caráter "stay-green".

\begin{tabular}{lrr}
\hline Fonte de variação & GL & Quadrado médio \\
\hline Ambiente (A) & 7 & $318,06^{* *}$ \\
Cruzamentos (C) & 249 & $2,59^{* *}$ \\
CGC Testadores (T) & 4 & $22,17^{* *}$ \\
CGC Linhagens (LI) & 49 & $7,29^{* *}$ \\
CEC (TxLI) & 196 & $1,02^{* *}$ \\
C x A & 1.743 & $0,46^{* *}$ \\
CGC (T) x A & 28 & $2,26^{* *}$ \\
CGC (LI) x A & 343 & $0,65^{* *}$ \\
CEC (TxLI) x A & 1.372 & $0,38^{* *}$ \\
Erro efetivo & 1.792 & 0,29 \\
CGC (T+LI) (\%) & & 69,06 \\
CEC (\%) & & 30,94 \\
CV (\%) & & 15,34 \\
\hline
\end{tabular}

*Significativo a $1 \%$ de probabilidade. grupo 2 que mais contribuíram para o aumento da expressão do caráter foram as linhagens 26 (43-03D), 5 (55-02D) e 16 (33-04D), com estimativas de -0,67, $-0,59$ e $-0,48$, respectivamente. Com relação às capacidades específicas de combinação, apenas $21,2 \%$ das 250 estimativas foram significativas ou altamente significativas, o que está de acordo com a maior contribuição da soma de quadrados decorrente dos efeitos aditivos observada na análise dialélica (Tabela 3).

As estimativas dos efeitos da capacidade geral de combinação proporcionam informações sobre a concentração de alelos predominantemente aditivos (Vencovsky \& Barriga, 1992), e seus efeitos têm sido de grande utilidade na indicação de genitores a serem utilizados em programas de melhoramento (Bordallo et al., 2005). Pode-se afirmar, portanto, que os melhores genitores do grupo das linhagens testadoras foram as linhagens 5 (L-46-10D) e 1 (L-08-05F). Quanto ao outro grupo de linhagens, as que apresentam maior capacidade geral de combinação foram as linhagens 26 (43-03D), 5 (55-02D) e 16 (33-04D).

Como a $\mathrm{CGC}_{\mathrm{T}}, \mathrm{CGC}_{\mathrm{LI}}$ e $\mathrm{CEC}_{\mathrm{TxLI}}$ foram altamente significativas, tanto os efeitos gênicos aditivos quanto os não aditivos estiveram envolvidos na expressão do caráter "stay-green". Porém, como houve predominância dos efeitos gênicos aditivos ( $\cong 70 \%)$, é possível realizar a seleção deste caráter durante o processo de obtenção de linhagens e, ainda, explorar alguma heterose durante o processo de obtenção de híbridos nos programas de melhoramento. Normalmente interessam aos melhoristas as combinações híbridas que apresentem estimativas de capacidades específicas de combinação favoráveis, e que pelo menos um dos genitores apresente maior capacidade geral de combinação.

Com base neste estudo, grupos de linhagens que possuam estimativas de capacidades gerais de combinação negativas e significativas, ou altamente significativas, podem ser utilizados para o desenvolvimento de variedades sintéticas visando à obtenção de linhagens com elevado "stay-green". Essas linhagens também poderão ser utilizadas como genitores em cruzamentos biparentais, para a formação de populações, ou em cruzamentos, para a obtenção de híbridos. 
Tabela 3. Estimativas das capacidades gerais de combinação das linhagens e testadores, capacidades específicas de combinação e respectivas significâncias para o caráter "stay-green".

\begin{tabular}{|c|c|c|c|c|c|c|}
\hline \multirow[t]{2}{*}{ Linhagem } & \multicolumn{5}{|c|}{ CEC } & \multirow[t]{2}{*}{ CGC } \\
\hline & Test 1 & Test 2 & Test 3 & Test 4 & Test 5 & \\
\hline$\overline{\text { Linh } 1}$ & $0,05^{\mathrm{ns}}$ & $-0,04^{\mathrm{ns}}$ & $0,12^{\mathrm{ns}}$ & $0,00^{\mathrm{ns}}$ & $-0,13^{\mathrm{ns}}$ & $-0,23^{*}$ \\
\hline Linh 2 & $0,25^{\mathrm{ns}}$ & $-0,25^{\mathrm{ns}}$ & $0,22^{\mathrm{ns}}$ & $-0,14^{\mathrm{ns}}$ & $-0,09^{\mathrm{ns}}$ & $0,32 * *$ \\
\hline Linh 3 & $-0,03^{\mathrm{ns}}$ & $0,18^{\mathrm{ns}}$ & $-0,21^{\mathrm{ns}}$ & $0,17^{\mathrm{ns}}$ & $-0,10^{\mathrm{ns}}$ & $-0,36^{* *}$ \\
\hline Linh 4 & $0,29 *$ & $-0,10^{\mathrm{ns}}$ & $-0,17^{\mathrm{ns}}$ & $-0,04^{\mathrm{ns}}$ & $0,02^{\mathrm{ns}}$ & $-0,30 * *$ \\
\hline Linh 5 & $0,61 * *$ & $-0,21^{\mathrm{ns}}$ & $-0,35^{*}$ & $-0,12^{\mathrm{ns}}$ & $0,07^{\mathrm{ns}}$ & $-0,59 * *$ \\
\hline Linh 6 & $0,62 * *$ & $-0,15^{\text {ns }}$ & $-0,41 * *$ & $0,13^{\mathrm{ns}}$ & $-0,19^{\text {ns }}$ & $-0,08^{\mathrm{ns}}$ \\
\hline Linh 7 & $0,28 *$ & $0,04^{\mathrm{ns}}$ & $0,01^{\mathrm{ns}}$ & $-0,37 * *$ & $0,04^{\mathrm{ns}}$ & $-0,16^{\mathrm{ns}}$ \\
\hline Linh 8 & $-0,34^{*}$ & $0,02^{\mathrm{ns}}$ & $0,24^{\mathrm{ns}}$ & $0,17^{\mathrm{ns}}$ & $-0,08^{\mathrm{ns}}$ & $-0,23 *$ \\
\hline Linh 9 & $-0,15^{\mathrm{ns}}$ & $-0,20^{\text {ns }}$ & $0,64 * *$ & $-0,19^{\text {ns }}$ & $-0,09^{\text {ns }}$ & $-0,09^{\mathrm{ns}}$ \\
\hline Linh 10 & $0,25^{\mathrm{ns}}$ & $0,22^{\mathrm{ns}}$ & $-0,10^{\mathrm{ns}}$ & $0,04^{\mathrm{ns}}$ & $-0,41 * *$ & $-0,10^{\mathrm{ns}}$ \\
\hline Linh 11 & $0,16^{\mathrm{ns}}$ & $0,01^{\mathrm{ns}}$ & $-0,35 * *$ & $0,20^{\mathrm{ns}}$ & $-0,02^{\mathrm{ns}}$ & $-0,41 * *$ \\
\hline Linh 12 & $-0,11^{\mathrm{ns}}$ & $0,15^{\mathrm{ns}}$ & $0,57 * *$ & $-0,24^{\mathrm{ns}}$ & $-0,36 * *$ & $0,07^{\mathrm{ns}}$ \\
\hline Linh 13 & $-0,05^{\mathrm{ns}}$ & $0,28 *$ & $0,30^{*}$ & $-0,29 *$ & $-0,25^{\mathrm{ns}}$ & $0,21 *$ \\
\hline Linh 14 & $0,06^{\mathrm{ns}}$ & $0,03^{\mathrm{ns}}$ & $0,22^{\mathrm{ns}}$ & $-0,29 *$ & $-0,02^{\mathrm{ns}}$ & $0,03^{\mathrm{ns}}$ \\
\hline Linh 15 & $-0,60 * *$ & $0,23^{\mathrm{ns}}$ & $0,32 *$ & $-0,32 *$ & $0,37 * *$ & $-0,22 *$ \\
\hline Linh 16 & $-0,53 * *$ & $0,14^{\mathrm{ns}}$ & $0,24^{\mathrm{ns}}$ & $-0,07^{\mathrm{ns}}$ & $0,22^{\mathrm{ns}}$ & $-0,48 * *$ \\
\hline Linh 17 & $0,16^{\mathrm{ns}}$ & $-0,26^{\text {ns }}$ & $-0,17^{\mathrm{ns}}$ & $-0,01^{\mathrm{ns}}$ & $0,28 *$ & $-0,08^{\mathrm{ns}}$ \\
\hline Linh 18 & $0,16^{\mathrm{ns}}$ & $0,03^{\text {ns }}$ & $-0,04^{\mathrm{ns}}$ & $0,16^{\mathrm{ns}}$ & $-0,31 *$ & $-0,08^{\mathrm{ns}}$ \\
\hline Linh 19 & $0,11^{\mathrm{ns}}$ & $0,34^{*}$ & $-0,20^{\mathrm{ns}}$ & $0,02^{\mathrm{ns}}$ & $-0,26^{\mathrm{ns}}$ & $-0,10^{\mathrm{ns}}$ \\
\hline Linh 20 & $0,26^{\mathrm{ns}}$ & $-0,10^{\mathrm{ns}}$ & $-0,04^{\mathrm{ns}}$ & $-0,11^{\mathrm{ns}}$ & $0,00^{\mathrm{ns}}$ & $0,45 * *$ \\
\hline Linh 21 & $-0,02^{\mathrm{ns}}$ & $-0,16^{\text {ns }}$ & $-0,17^{\text {ns }}$ & $0,34 *$ & $0,01^{\mathrm{ns}}$ & $0,45 * *$ \\
\hline Linh 22 & $0,19^{\text {ns }}$ & $-0,55 * *$ & $0,16^{\mathrm{ns}}$ & $0,18^{\mathrm{ns}}$ & $0,01^{\mathrm{ns}}$ & $0,59 * *$ \\
\hline Linh 23 & $0,21^{\mathrm{ns}}$ & $-0,07^{\mathrm{ns}}$ & $-0,21^{\mathrm{ns}}$ & $0,27^{\mathrm{ns}}$ & $-0,20^{\mathrm{ns}}$ & $0,18^{*}$ \\
\hline Linh 24 & $0,28^{*}$ & $-0,51 * *$ & $-0,03^{\mathrm{ns}}$ & $-0,14^{\mathrm{ns}}$ & $0,39 * *$ & $0,34 * *$ \\
\hline Linh 25 & $0,08^{\mathrm{ns}}$ & $-0,19^{\mathrm{ns}}$ & $-0,03^{\text {ns }}$ & $-0,02^{\mathrm{ns}}$ & $0,15^{\mathrm{ns}}$ & $0,03^{\mathrm{ns}}$ \\
\hline Linh 26 & $-0,35^{*}$ & $0,40 * *$ & $0,30 *$ & $-0,03^{\mathrm{ns}}$ & $-0,32 *$ & $-0,67 * *$ \\
\hline Linh 27 & $-0,05^{\mathrm{ns}}$ & $0,03^{\mathrm{ns}}$ & $0,21^{\mathrm{ns}}$ & $0,23^{\mathrm{ns}}$ & $-0,41 * *$ & $-0,06^{\mathrm{ns}}$ \\
\hline Linh 28 & $-0,01^{\mathrm{ns}}$ & $-0,08^{\mathrm{ns}}$ & $0,03^{\mathrm{ns}}$ & $0,37 * *$ & $-0,30 *$ & $-0,14^{\mathrm{ns}}$ \\
\hline Linh 29 & $0,20^{\text {ns }}$ & $0,01^{\text {ns }}$ & $-0,17^{\text {ns }}$ & $0,10^{\mathrm{ns}}$ & $-0,14^{\mathrm{ns}}$ & 0,25 ** \\
\hline Linh 30 & $-0,16^{\mathrm{ns}}$ & $0,09^{\mathrm{ns}}$ & $-0,07^{\mathrm{ns}}$ & $-0,12^{\mathrm{ns}}$ & $0,25^{\mathrm{ns}}$ & $0,31 * *$ \\
\hline Linh 31 & $-0,06^{\mathrm{ns}}$ & $0,20^{\mathrm{ns}}$ & $0,01^{\mathrm{ns}}$ & $-0,21^{\mathrm{ns}}$ & $0,06^{\mathrm{ns}}$ & $0,24 * *$ \\
\hline Linh 32 & $0,09^{\mathrm{ns}}$ & $-0,11^{\mathrm{ns}}$ & $-0,05^{\mathrm{ns}}$ & $0,04^{\mathrm{ns}}$ & $0,03^{\mathrm{ns}}$ & $0,32 * *$ \\
\hline Linh 33 & $-0,23^{\text {ns }}$ & $0,09^{\mathrm{ns}}$ & $0,14^{\mathrm{ns}}$ & $-0,27^{*}$ & $0,27^{*}$ & $0,57 * *$ \\
\hline Linh 34 & $-0,26^{\mathrm{ns}}$ & $0,07^{\mathrm{ns}}$ & $0,25^{\mathrm{ns}}$ & $-0,06^{\mathrm{ns}}$ & $-0,01^{\mathrm{ns}}$ & $0,27 * *$ \\
\hline Linh 35 & $-0,51 * *$ & $0,23^{\mathrm{ns}}$ & $0,20^{\mathrm{ns}}$ & $-0,15^{\mathrm{ns}}$ & $0,23^{\mathrm{ns}}$ & $-0,19^{*}$ \\
\hline Linh 36 & $-0,26^{\mathrm{ns}}$ & $-0,07^{\mathrm{ns}}$ & $0,04^{\mathrm{ns}}$ & $-0,36 * *$ & $0,65^{* *}$ & $0,44 * *$ \\
\hline Linh 37 & $-0,19^{\mathrm{ns}}$ & $0,38 * *$ & $-0,11^{\mathrm{ns}}$ & $-0,19^{\mathrm{ns}}$ & $0,12^{\mathrm{ns}}$ & $-0,06^{\mathrm{ns}}$ \\
\hline Linh 38 & $0,00^{\mathrm{ns}}$ & $0,05^{\mathrm{ns}}$ & $-0,10^{\mathrm{ns}}$ & $-0,07^{\mathrm{ns}}$ & $0,11^{\mathrm{ns}}$ & $-0,23 *$ \\
\hline Linh 39 & $-0,14^{\mathrm{ns}}$ & $0,26^{\mathrm{ns}}$ & $-0,04^{\mathrm{ns}}$ & $-0,26^{\mathrm{ns}}$ & $0,18^{\mathrm{ns}}$ & $0,14^{\mathrm{ns}}$ \\
\hline Linh 40 & $0,17^{\mathrm{ns}}$ & $-0,05^{\text {ns }}$ & $-0,38 * *$ & $0,36^{* *}$ & $-0,10^{\mathrm{ns}}$ & $-0,34 * *$ \\
\hline Linh 41 & $-0,41 * *$ & $-0,04^{\text {ns }}$ & $0,22^{\mathrm{ns}}$ & $0,35^{*}$ & $-0,12^{\text {ns }}$ & $-0,37 * *$ \\
\hline Linh 42 & $-0,13^{\mathrm{ns}}$ & $0,06^{\text {ns }}$ & $-0,07^{\text {ns }}$ & $-0,03^{\mathrm{ns}}$ & $0,16^{\mathrm{ns}}$ & $-0,03^{\mathrm{ns}}$ \\
\hline Linh 43 & $-0,09^{\mathrm{ns}}$ & $-0,23^{\mathrm{ns}}$ & $0,06^{\mathrm{ns}}$ & $0,24^{\mathrm{ns}}$ & $0,01^{\mathrm{ns}}$ & $-0,10^{\mathrm{ns}}$ \\
\hline Linh 44 & $-0,02^{\mathrm{ns}}$ & $0,12^{\mathrm{ns}}$ & $0,00^{\mathrm{ns}}$ & $0,11^{\mathrm{ns}}$ & $-0,21^{\mathrm{ns}}$ & $-0,08^{\mathrm{ns}}$ \\
\hline Linh 45 & $-0,06^{\mathrm{ns}}$ & $-0,06^{\mathrm{ns}}$ & $-0,13^{\mathrm{ns}}$ & $0,16^{\mathrm{ns}}$ & $0,09^{\mathrm{ns}}$ & $0,12^{\mathrm{ns}}$ \\
\hline Linh 46 & $0,29^{*}$ & $-0,35^{*}$ & $-0,29 *$ & $0,17^{\mathrm{ns}}$ & $0,18^{\mathrm{ns}}$ & $0,30 * *$ \\
\hline Linh 47 & $-0,24^{\mathrm{ns}}$ & $0,12^{\text {ns }}$ & $-0,03^{\mathrm{ns}}$ & $0,19^{\mathrm{ns}}$ & $-0,04^{\mathrm{ns}}$ & $-0,05^{\mathrm{ns}}$ \\
\hline Linh 48 & $0,28 *$ & $-0,11^{\mathrm{ns}}$ & $-0,52 * *$ & $0,01^{\mathrm{ns}}$ & $0,34^{*}$ & $0,33 * *$ \\
\hline Linh 49 & $-0,13^{\mathrm{ns}}$ & $0,17^{\text {ns }}$ & $-0,04^{\mathrm{ns}}$ & $-0,07^{\mathrm{ns}}$ & $0,08^{\mathrm{ns}}$ & $0,25 * *$ \\
\hline Linh 50 & $0,10^{\mathrm{ns}}$ & $-0,04^{\mathrm{ns}}$ & $-0,01^{\mathrm{ns}}$ & $0,13^{\text {ns }}$ & $-0,19^{\text {ns }}$ & $-0,40 * *$ \\
\hline$\overline{\mathrm{CGC}}$ & $-0,11^{*}$ & $0,16 * *$ & $-0,01 \mathrm{~ns}$ & $0,17 * *$ & $-0,21^{* *}$ & \\
\hline
\end{tabular}

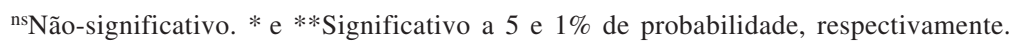




\section{Conclusões}

1. A contribuição dos efeitos gênicos aditivos é maior que a dos efeitos gênicos não aditivos na expressão fenotípica do caráter "stay-green" em milho.

2. O caráter "stay-green" interage significativamente com o ambiente, o que exige que a seleção desse caráter seja realizada com base em médias de experimentos com repetições em diversos ambientes.

\section{Agradecimentos}

Aos funcionários do Departamento de Genética da Escola Superior de Agricultura Luiz de Queiroz, pela contribuição na execução dos experimentos; à Coordenação de Aperfeiçoamento de Pessoal de Nível Superior e ao Conselho Nacional de Desenvolvimento Científico e Tecnológico, pelo apoio financeiro.

\section{Referências}

BEKAVAC, G.; PURAR, B.; STOJAKOVIC, M.; JOCKOVIC, D.J.; IVANOVIC, M.; NASTASIC, A. Genetic analysis of stay-green trait in broad-based maize populations. Cereal Research Communications, v.35, p.31-41, 2007.

BORDALLO, P.N.; PEREIRA, M.G.; AMARAL JÚNIOR, A.T.; GABRIEL, A.P.C. Análise dialélica de genótipos de milho doce e comum para caracteres agronômicos e proteína total. Horticultura Brasileira, v.23, p.123-127, 2005.

CAMARA, T.M.M. Mapeamento de QTLs de caracteres relacionados a tolerância ao estresse hídrico em milho. 2006. 177p. Tese (Doutorado) - Escola Superior de Agricultura Luiz de Queiroz, Piracicaba.

CARMO, S.L.M.; SANTOS, J.B.; HAGIWARA, W.E.; FERREIRA, J.L. Avaliação do "stay green" em famílias segregantes de feijão (Phaseolus vulgaris L.). Ciência e Agrotecnologia, v.31, p.953957, 2007.

CHAPMAN, S.C.; EDMEADES, G.O. Selection improves drought tolerance in tropical maize populations: II. Direct and correlated responses among secondary traits. Crop Science, v.39, p.13151324, 1999.

COCHRAM, W.G.; COX, S.M. Experimental designs. New York: Editora Wiley International, 1957. 611p.

CUKADAR-OLMEDO, B.; MILLER, J.F.; HAMMOND, J.J. Inheritance of the stay-green trait in sunflower. Crop Science, v.37, p.150-153, 1997.

DUVICK, D.N.; CASSMAN, K.G. Post-green revolution trends in yield potential of temperate maize in the North-Central United States. Crop Science, v.39, p.1622-1630, 1999.
EDMEADES, G.O.; BOLAÑOS, J.; CHAPMAN, S.C.; LAFITTE, H.R.; BÄNZIGER, M. Selection improves drought tolerance in tropical maize populations: I. Gains in biomass, grain yield and harvest index. Crop Science, v.39, p.1306-1315, 1999.

FERREIRA, D.F.; REZENDE, G.D.S.P.; RAMALHO, M.A.P. An adaptation of Griffing's Method IV of complete diallel cross analysis for experiments repeated in several environments. Brazilian Journal of Genetics, v.16, p.357-366, 1993.

GRIFFING, B. Concept of general and specific combining ability in relation to diallel crossing systems. Australian Journal of Biological Science, v.9, p.463-493, 1956.

JIANG, G.H.; HE, Y.Q.; XU, C.G.; LI, X.H.; ZHANG, Q. The genetic basis of stay-green in rice analyzed in a population of doubled haploid lines derived from an indica by japonica cross. Theoretical and Applied Genetics, v.108, p.688-698, 2004.

JOSHI, A.K.; KUMARI, M.; SINGH, V.P.; REDDY, C.M.; KUMAR, S.; RANE, J.; CHAND, R. Stay green trait: variation, inheritance and its association with spot blotch resistance in spring wheat (Triticum aestivum L.). Euphytica, v.153, p.5971, 2007.

SANTOS, M.F.; CÂMARA, T.M.M.; MORO, G.V.; COSTA, E.F.N.; SOUZA JÚNIOR, C.L. Responses to selection and changes in combining ability after three cycles of a modified reciprocal recurrent selection in maize. Euphytica, v.157, p.185194, 2007.

SAS Institute. SAS/STAT software. Cary: SAS, 1999. Version 8.2.

TOLLENAR, M.; WU, J. Yield improvement in temperate maize is attributable to greater stress tolerance. Crop Science, v.39, p.1597-1604, 1999.

THOMAS, H.; SMART, C. Crops that stay green. Annals of Applied Biology, v.123, p.193-219, 1993.

VENCOVSKY, R.; BARRIGA, P. Genética biométrica no fitomelhoramento. Ribeirão Preto: Sociedade Brasileira de Genética. 1992. 486p.

WU, H.; MATHESON, A.C. Analyses of half-diallel mating designs with missing crosses: theory and SAS program for testing and estimating GCA and SCA variance components. Silvae Genetica, v.50, p.265-271, 2001.

WU, H.X.; MATHESON, A.C. Analysis of half-diallel mating design with missing crosses: theory and SAS program for testing and estimating GCA and SCA fixed effects. Silvae Genetica, v.49, p.130-137, 2000.

YOKOMIZO, G.K.; VELLO, N.A. Coeficiente de determinação genotípica e de diversidade genética em topocruzamentos de soja tipo alimento com tipo grão. Pesquisa Agropecuária Brasileira, v.35, p.2223-2228, 2000.

ZHANG, Y.; KANG, M.S. DIALLEL-SAS: a program for Griffing's diallel methods. In: KANG, M.S. (Ed.). Handbook of formulas and software for plant geneticists and breeders. Binghamton: Food Products Press, 2003. p.1-20.

Recebido em 26 de outubro de 2007 e aprovado em 12 de fevereiro de 2008 